Books, videos, CD-ROMs, DVDs and any

other relevant items submitted for a review

in the $B D J$ should be addressed to: Kate

Maynard, Assistant Editor, British Dental

Journal, NPG, 4-6 Crinan Street, London,

N1 9XW
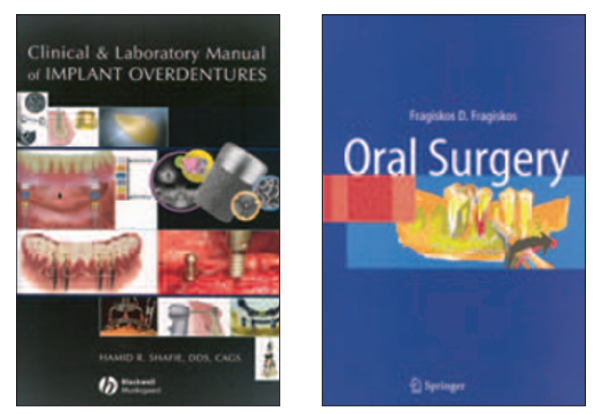

\section{Clinical and laboratory manual of implant overdentures}

\begin{tabular}{l} 
H. R. Shafie \\
UK: Blackwell Munksgaard \\
price $€ 75.00 ;$ pp 268 \\
ISBN 9780813808819 \\
\hline
\end{tabular}

This textbook, published by Blackwell, is devoted to implant overdentures, occupying a niche that few other books share. It is presented as a clinical and laboratory manual, and although it does contain some references to the literature, it is mainly of practical interest and focuses on planning issues, different systems and techniques.

It addresses various aspects of this treatment modality, starting with patient expectations, rationale and planning, clinical and laboratory prosthetic management, followed by selected implant systems and surgical considerations. This progression seems logical, developing an appreciation of the prosthetic requirements, prior to considering surgical planning. Implant systems considered in more detail include Strauman, Endopore, Maximus OS overdenture implants and ERA overdenture implants. There are complete chapters on spark erosion, occlusion, maintenance, and the core principles of a successful implant practice.

After classifying attachment types, based on resiliency, it focuses closely on stud and bar attachments. It describes some examples of the attachment systems available, applicable to various implant systems. Clinical and laboratory stages are described using a step-bystep approach, which despite being repetitive, allows the reader to extrapolate prosthetic techniques to other attachment systems.

This hardback book is visually appealing, with over 600 clinical images and schematic diagrams, in some cases superimposed to clarify points. The body of the text is divided into 17 chapters, each being on average
11 pages long. These are sub-divided clearly making it a very interesting and readable text, and suitable to use as a quick reference.

Overall, the book delivers a comprehensive and clear account of various aspects of implant-supported overdentures. It would be highly useful to both clinical and laboratory staff that are involved in the planning, surgical and prosthetic phases of treatment. Its clear and well illustrated format would make it appealing to those new to the subject matter, and its comprehensive content may attract the more experienced practitioner.

\section{P. Virdee}

\section{Oral surgery}

\section{F. D. Fragiskos \\ Germany: Springer \\ price $€ 191.50 ;$ pp 367 \\ ISBN 9783540251842}

This book was originally published in Greek by Professor Fragiskos, an Associate Professor in Oral and Maxillofacial Surgery, and has been translated into English by Helena Tsitsogianis. The aim is to provide practical guidance on various surgical techniques in the form of an atlas and the stated target audience is dental students, general dental practitioners and specialists. It is recognised by the author that this format limits the extent of theory behind the described techniques, reasoning that one picture is worth a thousand words.

There are 16 chapters covering many aspects of minor oral surgery, with particular emphasis on the basic principles. The first half of this book focuses on more common procedures from simple and surgical tooth extraction to management of odontogenic infections. The step-by-step illustrations give the reader a practical and visual guide on how to perform each surgical technique which is invaluable for dental students and young dentists. The latter half of the book concentrates on more advanced oral surgery, for example, salivary gland lesions, preprosthetic surgery and dental implants. These chapters are less detailed and provide more of an overview for experienced dental practitioners wishing to further their skills and professional development.

I was disappointed that oral premalignancy and cancer, medical emergencies and oral and maxillofacial trauma were not covered. This is a serious omission and an essential requisite to any textbook on oral surgery. Another major criticism is that some of the material is out of date or differs from what is taught and practised in the UK. In particular, the recommendations for steroid cover and antibacterial prophylaxis for infective endocarditis are not based on current clinical evidence; alternative treatments to surgical excision for lesions such as haemangiomas or salivary gland sialoliths are not considered; black silk sutures are used for closure of skin wounds and amalgam is the only retrograde restorative material discussed.

The presentation of this book makes it difficult to use as a quick reference or learning text. The chapters do not follow a structured programme and there would be great benefit in having an initial outline of key points, improved subheadings throughout and an end summary. Due to the translation from Greek, the text is excessively verbose and it could be better subtitled and paragraphed to ensure greater readability. Furthermore, the terminology used differs from that used in the UK, for example, apicoectomy instead of apicectomy, which is confusing.

Learning surgery from a written description is impossible and in the context of practical treatment, the use of clear diagrams and clinical pictures makes this book a useful adjunct to more theoretical textbooks. This book is beautifully illustrated and it provides an effective visual aid which will complement a clinician's professional learning. My overall opinion is that this book is lacking on detailed text, uses outdated 

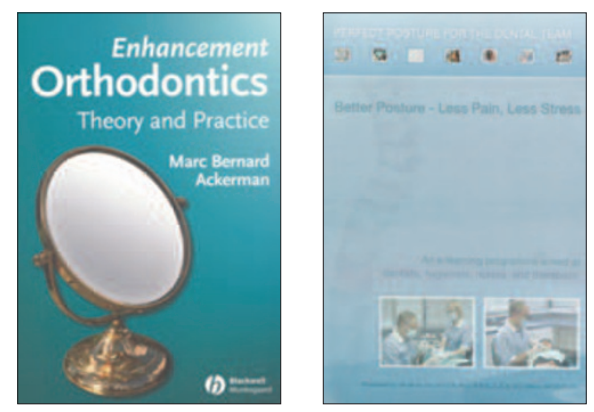

techniques and does not discuss more evidence based dentistry. It cannot rival similar, multi-author, specialist texts covering this speciality. I would only recommend the book as a supplemental text book for dental students and dental practitioners who require pictorial support for knowledge already acquired.

\section{K. Andrews}

\section{Enhancement orthodontics: theory and practice}

\author{
M. B. Ackerman \\ UK: Blackwell Munksgaard \\ price €39.99; pp 160
}

ISBN 9780813826233

This booklet reflects a different viewpoint on the provision of orthodontic treatment with respect to the aesthetic outcome of treatment. It is compiled of a well presented series of case reports supported with some references. There are very clear radiographic images indicating the flavour and quality of diagnostic imaging in the future. Within the text are clear and well documented templates for evaluating patients.

Whilst it can be accepted that some practitioners do not always heed the requests of patients, it should be part of the 'informed consent' process that can inform patients of what can and will be achieved. To imply that orthodontists do not evaluate the demands of their patients (reference to 'patient-centered' paradigms) is over-expressing what is actually what a good practitioner does. However, the booklet is well produced and has some good clinical material contained within. The cases do provoke some thought and it would be helpful for postgraduates to have looked at this text at some point during their careers; it is not a major reference text at 142 pages long but it is quite useful to stimulate thought.

\section{F. McDonald}

\section{Perfect posture for the dental team (CD-ROM)}

\section{E. Paul}

UK: Smile-on Ltd

price $€ 199.00+V A T$ or $£ 189.00$ for the online version. See www.smile-on.com

For over 25 years through his well known day courses, Ellis Paul has advocated the use of four-handed dentistry as an ergonomic solution for the dental team to minimise the risk of developing a musculoskeletal disorder.

At the start of this CD, members of the dental team (dentist, nurse and hygienist) are taken on a virtual tour of the 'Posture Practice'.

On arrival the team are told of the devastating effects back pain can result in if they do not work in this ergonomic manner. You could speak to fellow colleagues who are suffering with such a problem; however, what this CD does is educate you in how to overcome this problem by learning how to work comfortably in a tension-free manner.

The team are instructed in an easyto-follow step-by-step guide on how to incorporate and adopt this way of working in their current practice. The topics covered are: perfect posture; prerequisites; variables to keep good posture; soft tissue retraction; instrument handling and transfer; clinical procedures; and resources.
At the end of each section you can test yourself to ensure that the topics covered have sunk in. You can also log on and off the program as desired, starting again at the last point.

The photographs and video footage are of an excellent quality making it easy to readily see the new skills being demonstrated. The feature I like the most is how each chapter allows you to steadily build on the basics you have learnt from the previous chapter. By the end, you feel confident enough to have seen how to incorporate the ergonomic solutions, allowing you and your team to adopt a perfect working posture.

Finally, you can test yourself to ensure that you can apply all the principles of four-handed dentistry with day-to-day clinical scenarios.

At completion, you can look at the resources section. Here you can open files that have useful aide memoirs, ranging from the common postural problems you may find yourself working in and how to overcome them, to a section on how to keep your posture fit and healthy with core stability exercises.

Overall, a truly excellent CD that is a must for the student starting on the clinical aspect of their career to the long established practitioner who wants to work in an efficient, productive, tension free posture. The aim of this CD is to minimise the effects of developing a musculoskeletal problem and I feel every member of the dental team should have this knowledge added to their belt of key skills for working in primary dental care.

Well done to Ellis Paul and the team at Smile-on productions for an informative, easy to use educational CD.

R. Pilkington 